\title{
NUMERICAL SOLUTION OF FREE CONVECTIVE STRATIFIED FLUID FLOW OVER AN INFINITE VERTICAL POROUS PLATE WITH HALL EFFECT
}

\section{B. SHANKAR GOUD ${ }^{1 *}$, K. SUDHAKAR REDDY $^{2}$, P. SURESH $^{3} \&$ M.V. RAMANA MURTHY ${ }^{4}$}

\author{
${ }^{1}$ Department of Mathematics, JNTUH College of Engineering, Hyderabad, Telangana, State, India, -500085 \\ ${ }^{2}$ Department of Mechanical Engineering, Mahatma Gandhi Institute of Technology Gandipet, Hyderabad, Telangana State, \\ India, 500075 \\ ${ }^{3}$ Department of Mathematics, Chaitanya Bharathi Institute of Technology, Gandipet, Hyderabad, Telangana State, India, \\ 500075
}

${ }^{4}$ Department of Mathematics, Mahatma Gandhi Institute of Technology Gandipet, Hyderabad, Telangana State, India, 500075

ABSTRACT
The current paper focuses on the impacts of heat and mass transfer on the free convective flow through a porous medium
of stratified fluid. The flow is considered past a porous isothermal plate oriented vertically and disequilibrium with a time
dependent suction velocity. Finite element Galerkin method helped to derive numerical results for concentration field,
temperature field and velocity field. Variations in velocity, concentration, temperature distributions, Nusselt number and
skin friction due to the affecting physical quantities are graphically illustrated and discussed.
KEYWORDS: Stratified Flows, MHD Flows, Galerkian Method, Heat and Mass Transfer

Received: Jun 08, 2020; Accepted: Jun 28, 2020; Published: Aug 29, 2020; Paper Id.: IJMPERDJUN2020957

\section{INTRODUCTION}

Many industries, Chemical technology are primarily involved in heat and mass fluid flow where the main cause of flow is the continuous stretching of heated surfaces. Few scenarios of this type include wire and fibre coating, foodstuff dispensation, heat exchangers design and polymer procedures etc. The final result quality is dependent on flow and heat, mass transfer mechanism due to the stretching leading to a unidirectional orientation of extrudate Dharmaiah et al[1] deliberate the impacts of Hall current, thermophoresis and heat source on flow dissipative aligned convective flow about an inclined plate with perturbation analysis. Chamka [2] discussed the MHD flow of a uniformly stretched vertical permeable surface. Kandasamy et al.[3] obtained an estimated chemical reaction, heat and mass transfer solution on MHD flow over a vertical stretching surface with heat source and thermal stratification effect. Rashidi [4] developed the analytical solution with by using the DFM with the Pade's approximation on MHD boundary layer equations. Natural convection boundary layer flow of a double diffusive and revolving fluid past a vertical porous sheet was discussed by Reddy et al. [5]. Das and Mitra [6] studied on an unsteady MHD flow of mixed convective with suction \&mass transfer impacts on past an accelerated infinite vertical plate. The effect of the mass transfer on MHD flow and heat transfer over an oscillating porous plate via a porous medium was studied by Daset.al [7].Ibrahim and B.Reddy [8] talked about the impact of radiation and mass propagation on MHD natural convection movement along the stretching sheet, taking into consideration heat 
generation \& viscous dissipation. Jha et al [9] considered constant heat flux, mass transfer influences on the flow past an exponentially accelerated vertical plate. The influence of thermal radiation and magnetic field on an unsteady mixed convection flow and heat transfer issue from a porous vertical stretching layer was explored by Elbashbeshyet.al. [10]. Shankar Goud and Raja Shekar [11] studied Soret and radiation impact on mass transfer flow with heat generation and chemical reaction through a highly porous media. Ali Chamkha and Mansour [12] have studied the free convective heat and mass transfer, some impact of on an unsteady MHD flow over an infinite vertical plate. Satya Narayana et al. [13] examined Hall current impacts of free convective MHD flow on porous plate. Exact MHD-free convection solutions in heat-absorbing fluid flow past a flat plate with ramped wall temperature was examine by Nandkeolyar et.al [14]. The results of heat and thermal radiation on an unsteady MHD free convection flow through an infinite vertical plate of thermal diffusion and diffusion thermo was studied by Raju et.al [15]. B.S Goud et.al[16] examine the MHD flow on inclined plate with mass transfer effect through porous medium in the existence of the temperature and thermal radiation. B.S Goud [17] investigated on stretching with slip boundary conditions with thermal radiation influence on MHD stagnation point flow.BSGoudet.al [18] investigated on MHD flow of free convection flow with radiatve effect over an vertical surface over a porous medium in the influence of the temperature.

Considering the above, this paper has focussed on results of Hall current\& mass transfer on stratified fluid's free convective flow through a porous medium. The study is made past an unstable isothermal vertical porous plate where the fluctuation is time dependent suction velocity. Galerkin finite element procedure is applied to derive numerical solution for concentration, temperature field and velocity field. The results of several physical factors on the velocity temperature and concentration fields, Skin friction and Nusselt number are considered by means of graphs in detail.

\section{MATHEMATICAL FORMULATION}

Consider the free convective movement through an infinite vertical, porous, isothermal plate with time-dependent suction on the plate of an incompressible, electrically conducting viscous, stratified fluid through the porous medium. It is assumed that the flow is in $\mathrm{x}$-direction that is taken in the upward direction along the vertical plate and that the $y$-axis is taken to the plate. A uniform magnetic field $B=B_{o} e^{-\alpha y}$ is implemented to the plate in the normal direction and the suction velocity $v=-v_{o}\{1+\varepsilon f(t)\}$ being a function of time and the pressure gradient being negligible. The present influence of the Hall current taken into consideration.

Consider the influence of the density difference with temperature only in term of body force but it is assumed that all fluid characteristics are constant. In the act upon of density variation, different terms of the energy equation and variety of expansion coefficient with temperature are viewed as irrelevant. The free convection streams are in presence because of temperature distinction. Hence, using the above postulations, the governing equations for the flow are:

Momentum equation

$\left(\frac{\partial u}{\partial t}+v \frac{\partial u}{\partial y}\right)=\frac{\mu}{\rho} \frac{\partial}{\partial y}\left(\frac{\partial u}{\partial y}\right)-\frac{\sigma B_{o}^{2}}{\rho\left(1+m^{2}\right)} u+g \beta\left(T-T_{\infty}\right)+g \beta^{*}\left(C-C_{\infty}\right)-\frac{\mu}{\rho k} u$

Temperature equation

$\left(\frac{\partial T}{\partial t}+v \frac{\partial T}{\partial y}\right)=\frac{k}{\rho C_{p}} \frac{\partial}{\partial y}\left(\frac{\partial T}{\partial y}\right)$

Concentration equation 


$$
\left(\frac{\partial C}{\partial t}+v \frac{\partial C}{\partial y}\right)=D_{1} \frac{\partial}{\partial y}\left(\frac{\partial C}{\partial y}\right)+D_{2} \frac{\partial}{\partial y}\left(\frac{\partial T}{\partial y}\right)
$$

Here we are assuming the $\operatorname{den} \operatorname{sity}(\rho)$, $\operatorname{viscosity}(\mu)$, thermal conductivity $(\mathrm{K})$, volumetric coefficient $\operatorname{expansion}(\beta)$ and species coefficient $\left(\beta^{*}\right)$ satisfy exponential law. Namely $\rho=\rho_{o} e^{-a y}, \mu=\mu_{o} e^{-a y}, k=k_{t} e^{-a y}, \beta=$ $\beta_{o} e^{-a y}$ and $\beta^{*}=\beta_{o}^{*} e^{-a y}$ where $a$ is a stratification factor.

Applying the above values in equation (1) - (3), we get

$$
\begin{aligned}
& \left(\frac{\partial u}{\partial t}-v_{o}\{1+\varepsilon f(t)\} \frac{\partial u}{\partial y}\right)=v \frac{\partial}{\partial y}\left(\frac{\partial u}{\partial y}\right)-\alpha v \frac{\partial u}{\partial y}-\frac{\sigma B_{o}^{2}}{\rho\left(1+m^{2}\right)} u+g \beta\left(T-T_{\infty}\right)+g \beta^{*}\left(C-C_{\infty}\right)-\frac{v}{k} u \\
& \left(\frac{\partial T}{\partial t}-v_{o}\{1+\varepsilon f(t)\} \frac{\partial T}{\partial y}\right)=\frac{k}{\rho C_{p}} \frac{\partial}{\partial y}\left(\frac{\partial T}{\partial y}\right) \\
& \left(\frac{\partial C}{\partial t}-v_{o}\{1+\varepsilon f(t)\} \frac{\partial C}{\partial y}\right)=D_{1} \frac{\partial}{\partial y}\left(\frac{\partial C}{\partial y}\right)+D_{2} \frac{\partial}{\partial y}\left(\frac{\partial T}{\partial y}\right)
\end{aligned}
$$

The boundary conditions are

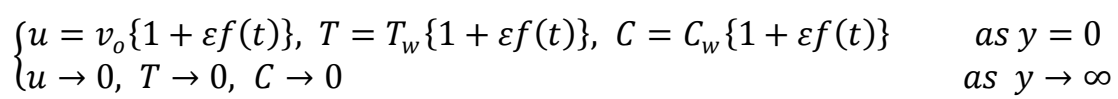

Now introducing the non- dimensional variables:

$$
\bar{y}=\frac{y v_{o}}{v}, \bar{u}=\frac{u}{v_{o}}, \bar{t}=\frac{t v_{o}^{2}}{v}, \theta=\frac{T-T_{\infty}}{T_{w}-T_{\infty}}, C=\frac{C-C_{\infty}}{C_{w}-C_{\infty}}, \bar{K}=\frac{K v_{o}^{2}}{v}
$$

Using the above non dimensional variable into the equation (4) -(6), we get

$$
\begin{aligned}
& \frac{\partial u}{\partial t}-\{1+\varepsilon f(t)\} \frac{\partial u}{\partial y}=\frac{\partial}{\partial y}\left(\frac{\partial u}{\partial y}\right)-S \frac{\partial u}{\partial y}-\left(\frac{M^{2}}{\left(1+m^{2}\right)}+\frac{1}{K}\right) u+G r \theta+G c \mathrm{C} \\
& \frac{\partial T}{\partial t}-\{1+\varepsilon f(t)\} \frac{\partial T}{\partial y}=\frac{1}{P r} \frac{\partial}{\partial y}\left(\frac{\partial T}{\partial y}\right) \\
& \frac{\partial C}{\partial t}-\{1+\varepsilon f(t)\} \frac{\partial C}{\partial y}=\frac{1}{S c} \frac{\partial}{\partial y}\left(\frac{\partial C}{\partial y}\right)+S_{0} \frac{\partial}{\partial y}\left(\frac{\partial T}{\partial y}\right)
\end{aligned}
$$

Where

$$
\begin{gathered}
S=\frac{v \alpha}{v_{o}}, G r=\frac{g \beta v\left(T_{w}-T_{\infty}\right)}{v_{0}^{3}}, G c=\frac{g \beta^{*} v\left(C-C_{\infty}\right)}{v_{0}^{3}}, M^{2}=\frac{\sigma v \beta_{o}^{2}}{\rho v_{0}^{2}}, \operatorname{Pr}=\frac{\mu C_{p}}{K}, S c=\frac{v}{D^{\prime}} \\
S_{o}=\frac{D_{1}}{v}\left(\frac{C-C_{\infty}}{C_{w}-C_{\infty}}\right),
\end{gathered}
$$

The corresponding non dimensional conditions are

$$
\begin{aligned}
& u=1+\varepsilon f(t), \theta=1+\varepsilon f(t), C=1+\varepsilon f(t) \quad \text { as } y=0 \\
& u \rightarrow 0, \theta \rightarrow 0, C \rightarrow 0 \quad \text { as } y \rightarrow \infty
\end{aligned}
$$

To solve the problem, we consider the case when the suction velocity decreases the function of the time exponentially. Hence we assume $f(t)=e^{-n t}$

Substituting equation (12) in to the equation (8) - (10), we get 


$$
\begin{aligned}
& \frac{\partial u}{\partial t}-\left\{1+\varepsilon e^{-n t}\right\} \frac{\partial u}{\partial y}=\frac{\partial}{\partial y}\left(\frac{\partial u}{\partial y}\right)-S \frac{\partial u}{\partial y}-\left(\frac{M^{2}}{\left(1+m^{2}\right)}+\frac{1}{K}\right) u+G r \theta+G c \mathrm{C} \\
& \frac{\partial T}{\partial t}-\left\{1+\varepsilon e^{-n t}\right\} \frac{\partial T}{\partial y}=\frac{1}{P r} \frac{\partial}{\partial y}\left(\frac{\partial T}{\partial y}\right) \\
& \frac{\partial C}{\partial t}-\left\{1+\varepsilon e^{-n t}\right\} \frac{\partial C}{\partial y}=\frac{1}{S c} \frac{\partial}{\partial y}\left(\frac{\partial C}{\partial y}\right)+S_{0} \frac{\partial}{\partial y}\left(\frac{\partial T}{\partial y}\right)
\end{aligned}
$$

The changed boundary conditions are:

$$
\begin{array}{ll}
u=1+\varepsilon e^{-n t}, \theta=1+\varepsilon e^{-n t}, C=1+\varepsilon e^{-n t} & \text { as } y=0 \\
u \rightarrow 0, \quad \theta \rightarrow 0, \quad C \rightarrow 0 & \text { as } y \rightarrow \infty
\end{array}
$$

\section{SOLUTION OF THE PROBLEM}

By applying the efficient Galerk infinite element method to the equation (13) over the two nodded elemente $\left(y_{j} \leq\right.$ $y \leq y k$ which is linear and implementing the Crank-Nikolson variational technique, obtain the following

$$
\begin{aligned}
& \int_{y_{j}}^{y_{j}}\left\{\xi^{(e)}\left[\frac{\partial^{2} u^{(e)}}{\partial y^{2}}-S \frac{\partial u^{(e)}}{\partial y}+B \frac{\partial u^{(e)}}{\partial y}-\frac{\partial u^{(e)}}{\partial t}-\zeta u^{(e)}+G r \theta+G c C\right]\right\} \\
& \int_{y_{j}}^{y_{j}}\left\{\xi^{(e)}\left[\frac{\partial^{2} u^{(e)}}{\partial y^{2}}+(B-S) \frac{\partial u^{(e)}}{\partial y}-\frac{\partial u^{(e)}}{\partial t}-\zeta u^{(e)}+R_{1}\right]\right\}
\end{aligned}
$$

Here $R_{1}=G r \theta+G c C, B=1+\varepsilon e^{-n t}, \zeta=\left(\frac{M^{2}}{1+m^{2}}+\frac{1}{K}\right)$

Integrating Eqn(16) the initial term by method of by parts

$$
\left\{\xi^{(e)} \frac{\partial u^{(e)}}{\partial y}\right\}_{y_{j}}^{y_{k}}-\int_{y_{j}}^{y_{k}} \frac{\partial \xi^{(e)}}{\partial y} \frac{\partial u^{(e)}}{\partial y} d y-(B-S) \int_{y_{j}}^{y_{k}} \xi^{(e)} \frac{\partial u^{(e)}}{\partial y} d y-\int_{y_{j}}^{y_{k}} \xi^{(e)} \frac{\partial u^{(e)}}{\partial t} d y-\zeta \int_{y_{j}}^{y_{k}} \xi^{(e)} u^{(e)} d y+\int_{y_{j}}^{y_{k}} \xi^{(e)} R_{1} d y=0
$$

And, omitting the first term if equation (17), we achieve the following part

$$
\int_{y_{j}}^{y_{k}} \frac{\partial \xi^{(e)}}{\partial y} \frac{\partial u^{(e)}}{\partial y} d y+(S-B) \int_{y_{j}}^{y_{k}} \xi^{(e)} \frac{\partial u^{(e)}}{\partial y} d y+\int_{y_{j}}^{y_{k}} \xi^{(e)} \frac{\partial u^{(e)}}{\partial t} d y+\zeta \int_{y_{j}}^{y_{k}} \xi^{(e)} u^{(e)} d y \int_{y_{j}}^{y_{k}} \xi^{(e)} R_{1} d y
$$

Finite element model can be obtained by replacing finite element approximation over the two linear nodded elements $(e)\left(y_{j} \leq y \leq y_{k}\right)$ from equation (17) of the form:

$$
u^{(e)}=N^{(e)} \xi^{(e)}, \text { here } \psi^{(e)}=\left[\xi_{j} \xi_{k}\right], N^{(e)}=\left[u_{j} u_{k}\right]^{T}
$$

Where $u_{j} u_{k}$ are the components of the velocity factors at ${ }^{t h}$ and $k^{t h}$ nodes of the distinctive element $(e),\left(y_{j} \leq\right.$ $y \leq y k$ and the basis functions can defined as follows. $\xi_{j}=\frac{y_{k}-y}{y_{k}-y_{j}}, \xi_{k}=\frac{y-y_{j}}{y_{k}-y_{j}}$. Substituting (19) in equ.(18) the following is obtained 


$$
\frac{1}{l^{(e)}}\left[\begin{array}{rr}
1 & -1 \\
-1 & 1
\end{array}\right]\left[\begin{array}{l}
u_{j} \\
u_{k}
\end{array}\right]+\frac{l^{(e)}}{6}\left[\begin{array}{ll}
2 & 1 \\
1 & 2
\end{array}\right]\left[\begin{array}{l}
\dot{u}_{j} \\
\dot{u}_{k}
\end{array}\right]+\frac{(S-B)}{2 l^{(e)}}\left[\begin{array}{ll}
-1 & 1 \\
-1 & 1
\end{array}\right]\left[\begin{array}{l}
u_{j} \\
u_{k}
\end{array}\right]+\frac{\zeta l^{(e)}}{6}\left[\begin{array}{ll}
2 & 1 \\
1 & 2
\end{array}\right]\left[\begin{array}{l}
u_{j} \\
u_{k}
\end{array}\right]=\frac{R_{1} l^{(e)}}{2}\left[\begin{array}{l}
1 \\
1
\end{array}\right]
$$

Where ' $\bullet$ ' denote the diff..w. respect to time, and the length of the element is $l^{(e)}=y_{k}-y_{j}$.Assembling two successive components with element equations $\left(y_{j-1} \leq y \leq y_{i}\right)$ and $\left(y_{i} \leq y \leq y_{i+1}\right)$ by inter-element connectivity and obtained the following

$$
\frac{1}{6}\left[\begin{array}{lll}
2 & 1 & 0 \\
1 & 4 & 1 \\
0 & 1 & 2
\end{array}\right]\left[\begin{array}{c}
u_{i-1}^{\cdot} \\
\dot{u}_{i} \\
u_{i+1}^{\cdot}
\end{array}\right]+\frac{1}{l^{(e)^{2}}}\left[\begin{array}{ccc}
1 & -1 & 0 \\
-1 & 2 & -1 \\
0 & -1 & 1
\end{array}\right]\left[\begin{array}{c}
u_{i-1} \\
u_{i} \\
u_{i+1}
\end{array}\right]+\frac{(S-B)}{2 l^{(e)}}\left[\begin{array}{ccc}
-1 & 1 & 0 \\
-1 & 0 & 1 \\
0 & -1 & 1
\end{array}\right]\left[\begin{array}{c}
u_{i-1} \\
u_{i} \\
u_{i+1}
\end{array}\right]+\frac{\zeta}{6}\left[\begin{array}{lll}
2 & 1 & 0 \\
1 & 4 & 1 \\
0 & 1 & 2
\end{array}\right]\left[\begin{array}{c}
u_{i-1} \\
u_{i} \\
u_{i+1}
\end{array}\right]=\frac{R_{1}}{2}\left[\begin{array}{l}
1 \\
2 \\
1
\end{array}\right]
$$

The following differential methods are performed when the resulting section of the node "i"is equal to zero:

$$
\frac{1}{6}\left[\dot{u}_{i-1}+4 \dot{u_{i}}+\dot{u}_{i+1}\right]+\frac{1}{h^{2}}\left[-u_{i-1}+2 u_{i}-u_{i+1}\right]+\frac{(S-B)}{2 h}\left[-u_{i-1}+u_{i+1}\right]+\frac{\zeta}{6}\left[u_{i-1}+4 u_{i}+u_{i+1}\right]=R_{1}---(21)
$$

The following formulas are generated by means of the trapezoid and formula (18) in Crank - Nicholson method:

$$
\begin{aligned}
& (2-3(S-B) r h-6 r+\zeta k) u_{i-1}^{j+1}+(4 \zeta k+12 r+8) u_{i}^{j+1}+(3(S-B) r h+6 r-\zeta k+2) u_{i+1}^{j+1}= \\
& (2+3(S-B) r h+6 r-\zeta k) u_{i-1}^{j}+(-4 \zeta k-12 r+8) u_{i}^{j}+(2-3(S-B) r h+6 r-\zeta k) u_{i+1}^{j}+12 R_{1} k \\
& {\left[A_{1} A_{2} A_{3}\right]_{1 \times 3} \cdot\left[u_{i-1}^{n+1} u_{i}^{n+1} u_{i}^{n+1}\right]_{3 \times 1}^{T}-\left[A_{4} A_{5} A_{6}\right]_{1 \times 3} \cdot\left[u_{i-1}^{n+1} u_{i}^{n+1} u_{i}^{n+1}\right]_{3 \times 1}^{T}=R^{*}}
\end{aligned}
$$

$$
A_{1}=2-3(S-B) r h+\zeta k-6 r, A_{2}=8+4 \zeta k+12 r, A_{3}=2+3(S-B) r h+6 r-\zeta k
$$

Where $A_{4}=2+3(S-B) r h-\zeta k+6 r, A_{5}=8-4 \zeta k-12 r, A_{6}=2-3(S-B) r h+6 r-\zeta k$

$$
12 R_{1} k=12 k\left(\operatorname{Gr} \theta_{i}^{j}+G m C_{i}^{j}\right)
$$

The following formulas were obtained by using the Galerk infinite element scheme for equation (12) - (13) in the same way and

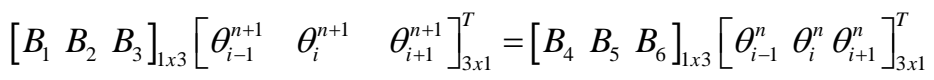

$$
\begin{aligned}
& {\left[\begin{array}{lll}
C_{1} & C_{2} & C_{3}
\end{array}\right]_{1 \times 3}\left[\begin{array}{lll}
C_{i-1}^{n+1} & C_{i}^{n+1} C_{i+1}^{n+1}
\end{array}\right]_{3 \times 1}^{T}-\left[\begin{array}{lll}
C_{4} & C_{5} & C_{6}
\end{array}\right]_{1 \times 3}\left[C_{i-1}^{n} C_{i}^{n} C_{i+1}^{n}\right]_{3 x 1}^{T}=R^{* *}} \\
& B_{1}=-6 r+2 \operatorname{Pr}+3 r h B \operatorname{Pr}, B_{2}=12 r+8 \operatorname{Pr}, B_{3}=2 \operatorname{Pr}-6 r-3 r h B \operatorname{Pr} \text {, } \\
& B_{4}=6 r+2 \operatorname{Pr}-3 r h B \operatorname{Pr}, B_{5}=-12 r+8 \operatorname{Pr}, B_{6}=-6 r+2 \operatorname{Pr}-3 r h B \operatorname{Pr},
\end{aligned}
$$

Where $C_{1}=-6 r+3 r h B S c+2 S c, C_{2}=12 r+8 S c, C_{3}=-6 r+2 S c-3 r h B S c$,

$$
\begin{aligned}
& C_{4}=-6 r+2 S c-3 r h B S c r, C_{5}=-12 r+8 S c, C_{6}=6 r+2 S c+3 r h B S c, \\
& R^{* *}=12 k S c S o\left(\frac{\partial^{2} \theta_{i}}{\partial y_{i}^{2}}\right)^{2}, r=\frac{h}{k^{2}}
\end{aligned}
$$

Here $h=0.1 \& k=0.01$ is the mesh dimensions in the direction of $y$-and $t$ - respectively, and $(i, n)$ mentioned to the space $\&$ time. In equation (21), (22) and (23), as suming $i=1(1) m$ and using (14), we obtained the set of equations as 


$$
A_{i} \cdot X_{i}=B_{i} i=1(1) m
$$

Here $\left(A_{i}\right)_{m \times m},\left(X_{i}\right)_{m \times 1},\left(B_{i}\right)_{m \times 1}$ are the matrices. The solutions of the Equ.(15) are obtained by using Thomas algorithm for the flow fields. Numerical calculations are achieved by running the MATLAB-program until the steady state is reached by taking the smaller values of $h, k$ and found that there is no significant variation in the values of flow distributions. Therefore, the process of finite element technique is consistent and convergent. The results are calculated and presented graphically for different parameters. Certain physical quantities of importance can be easily calculated for this problem, namely the Skin friction coefficient at the plate at $y=0$ is given by is $\tau=\left(\frac{\partial u}{\partial y}\right)_{y=0}$ The heat transfer rate in terms of Nusselt number $(N u)$ at the vertical plate at $y=0$ is given by is $\left(\frac{\partial \theta}{\partial y}\right)_{y=0}$. The mass transfer rate quantity at the vertical plate is $\left(\frac{\partial c}{\partial y}\right)_{y=0}$

\section{RESULTS AND DISCUSSIONS}

Graphical evaluation for the physical derived quantities i.e. temperature, velocity and concentration is done for varying Hall parameter $(m)$, Hartmann number $(M)$, Grash of number $(G r)$, Modified Grash of number (Gc), Soret number (So), Schmidt number $(S c)$, Prandtl number (Prif); and the frequency parameter $(n)$,Stratification factor $(S)$,Skin friction, Nusselt number, and Sherwood number.

Figure 1 demonstrates the influence on temperature distribution of the Prandtl number. It is found the temperature curves are diminishes with enhance of $\operatorname{Pr}$ values. Figure 2 present the behaviour of the Prandtl number on the concentration distribution. Clearly, Concentration curves keep rising in $\operatorname{Pr}$ Change. Soret number So leads to increased concentration level. Figure 3 presents the impact of Soret number on the concentration profile. It is observed that the concentration curves are drops with an increase of So.Figure5 defined the impact on the velocity profile of the Prandtl number(Pr),and rise in $\operatorname{Pr}$ causes the fluid boundary layer to decrease. Fig. 4 and 6present the impact of Schmidt number (Sc)on concentration and velocity fields. It can be seen that observed that, concentration declines and the velocity increases with increasing 'Sc'. Figure 7 describes the influence on the velocity curves an increase of Soret number. It is observer that the velocity rises with an enhancing the So. Similarly figure8 reports the velocity distribution of several values of Hartmann number $(M)$. It is known that the velocity profile rises as a consequence of the parameters being increased. Figure 9 indicates that the higher values of Hall parameter $(\mathrm{m})$ substantially increase the velocity curves. Figure 10 demonstrate that the behaviour of the Grashof number on the velocity curves. It It is introduced that the flow velocity curves are enhanced by an improvement in Gr.Figure11 illustrate the outcome of Modify Grashof number (Gc) on the field of velocity. An improvement in Gc obviously leads to a higher velocity. Figure 12 demonstrates the velocity profile impact of Stratification factor $(\mathrm{S})$. The velocity curves are found to increase insignificantly with rising values of S. The result on Nusselt number is given in Fig. 13-14. With an increment in time $t$ or the frequency parameter n, Nusselt number is noticed to decrease. Alternatively, the behaviour of increase in Nusselt number is found with an increase in Pr.

\section{CONCLUSIONS}

In this problem of solution, the influence of Hall current impacts on free convective stratified fluid flow over an infinite vertical permeable plate is analyzed. Changed values of non-dimensional parameters are calculated numerically. A 
method of Galerkin finite elements was used to evaluate the solution for concentration, velocity, temperature, Sherwood number, skin friction, and Nusselt number. The decisions are as follows:

- Both velocity and temperature diminishes with enhance in $\operatorname{Pr}, S c$ and $M$.

- Enhances the velocity with an increase in $\mathrm{m}, S o, G r, G c$ and $S$.

- With an increase of $P r$, So Concentration gains and decreases with raise in $S c$.

- With time $t$ increases Nusselt number diminishes and increases with an increase of Pr.

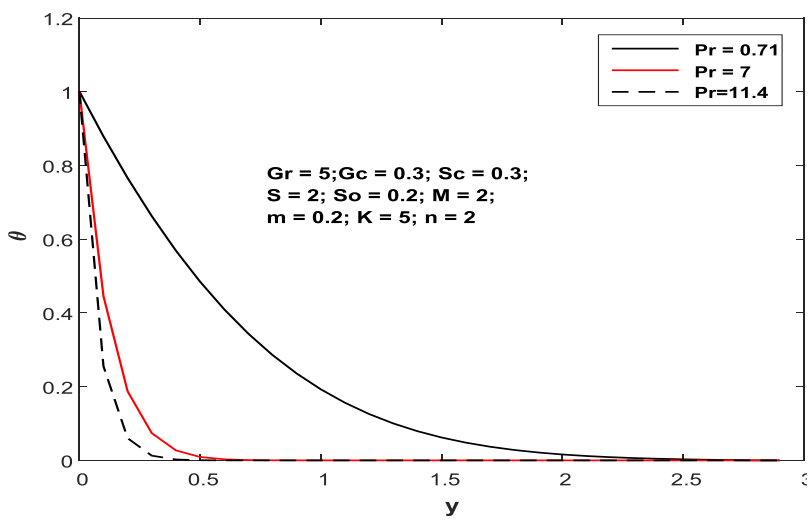

Figure 1: Effect of $\operatorname{Pr} \mathbf{V} / \mathrm{S}$ Temperature Field.

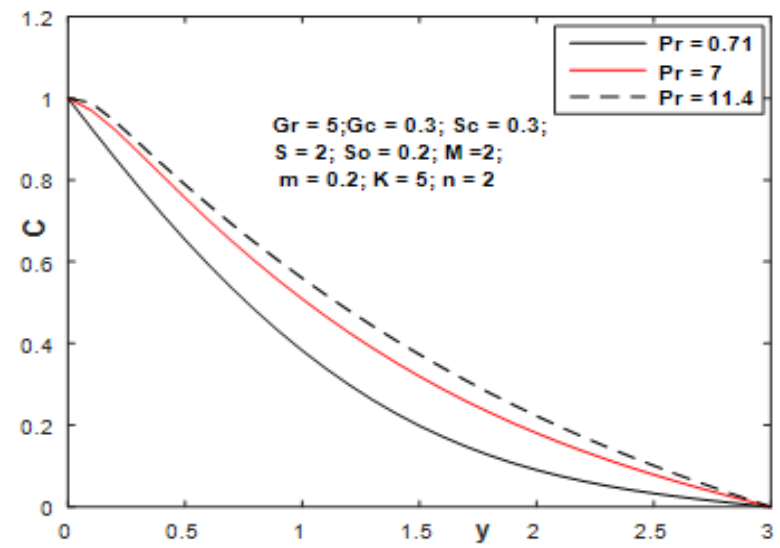

Figure 2: Changes of $\operatorname{Pr}$ V/S Concentration Field.

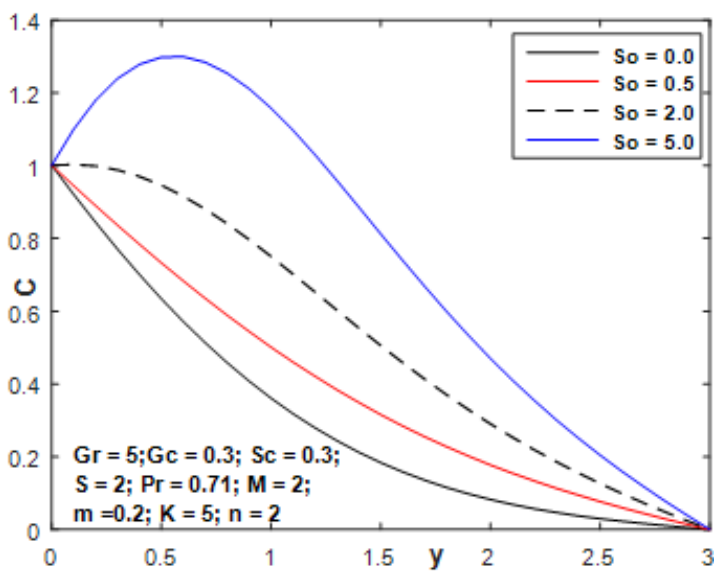

Figure 3: Outcomes of $S o$ V/S Concentration Field. 


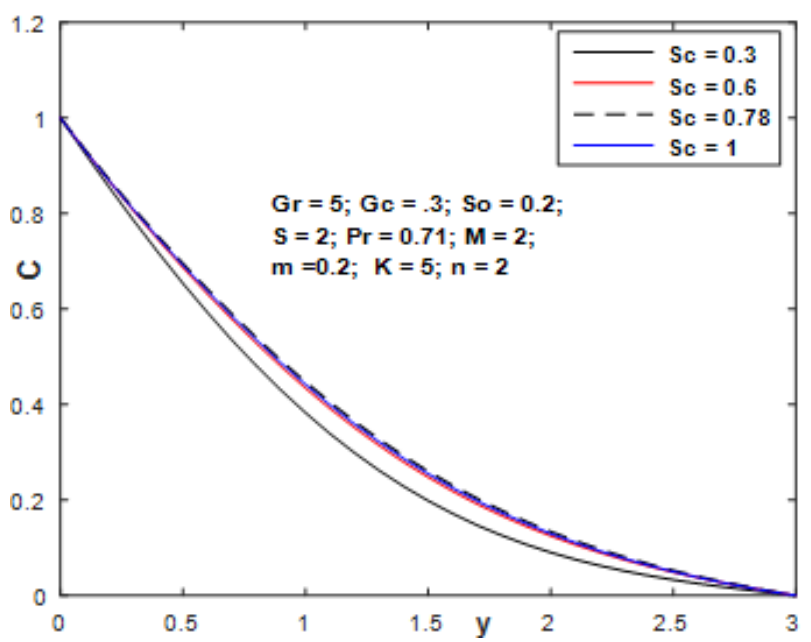

Figure 4: Impact of $S c$ V/S Concentration Field.

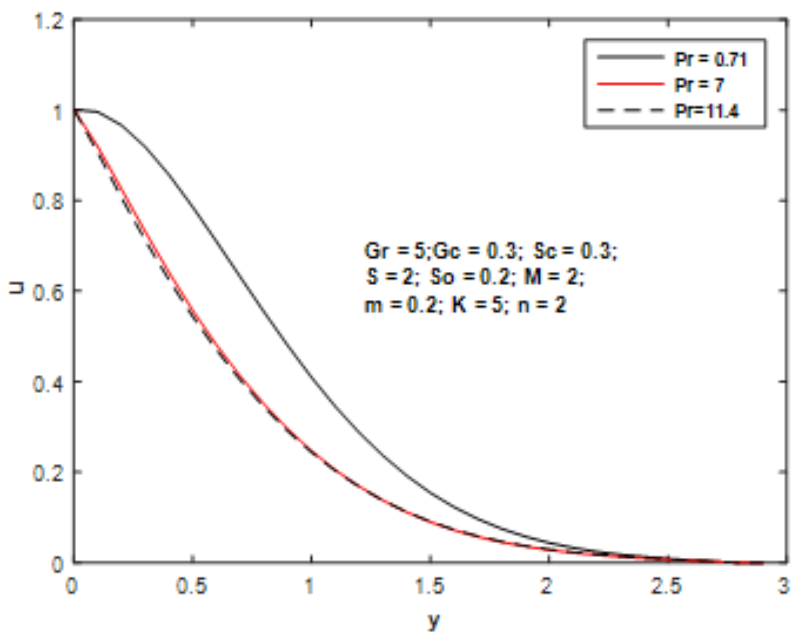

Figure 5: Changes of Velocity field V/S Pr.

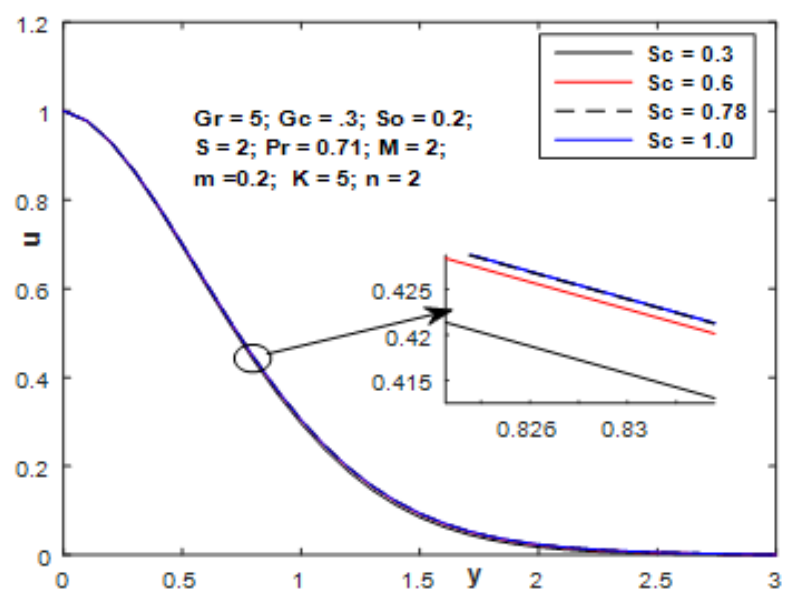

Figure 6: Changes Velocity field V/S $S c$. 


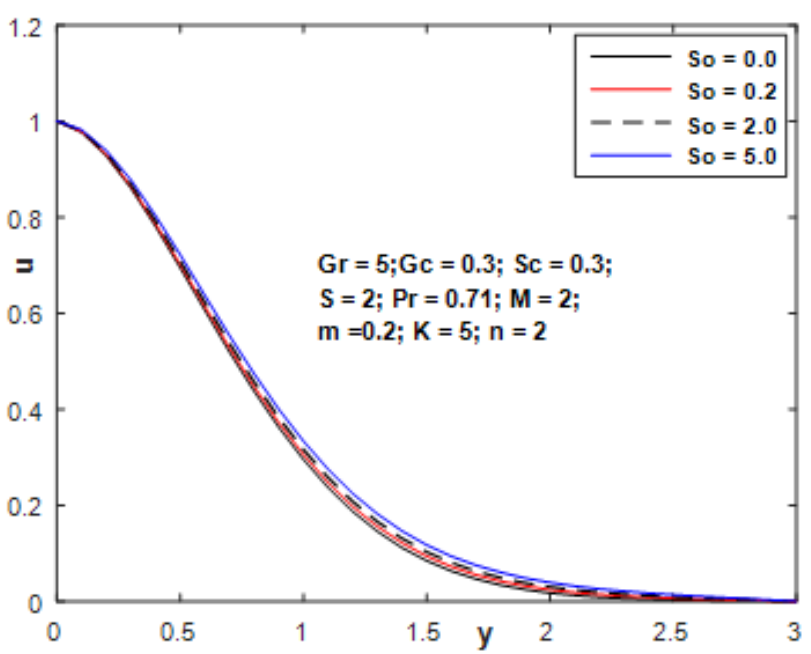

Figure 7: Changes Velocity field V/S So.

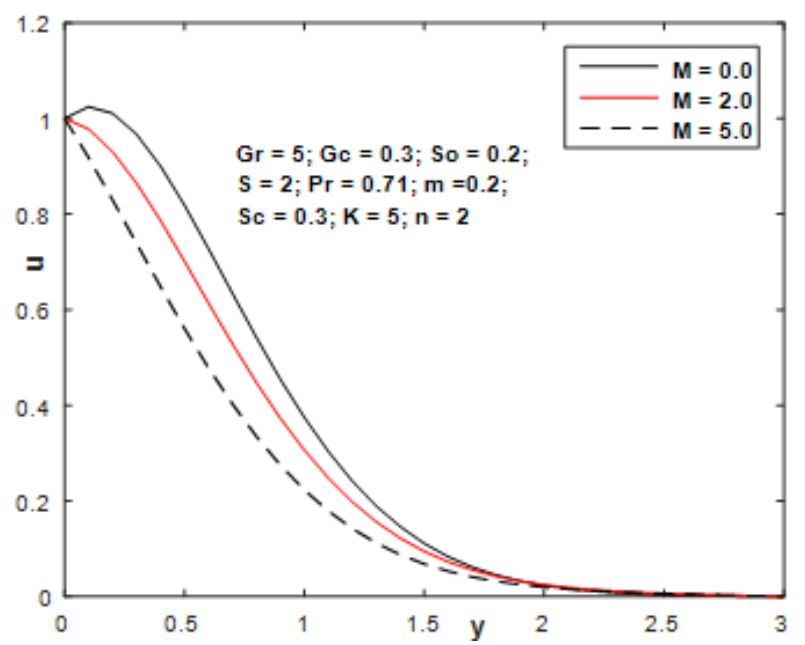

Figure 8: Changes Velocity field V/S $M$.

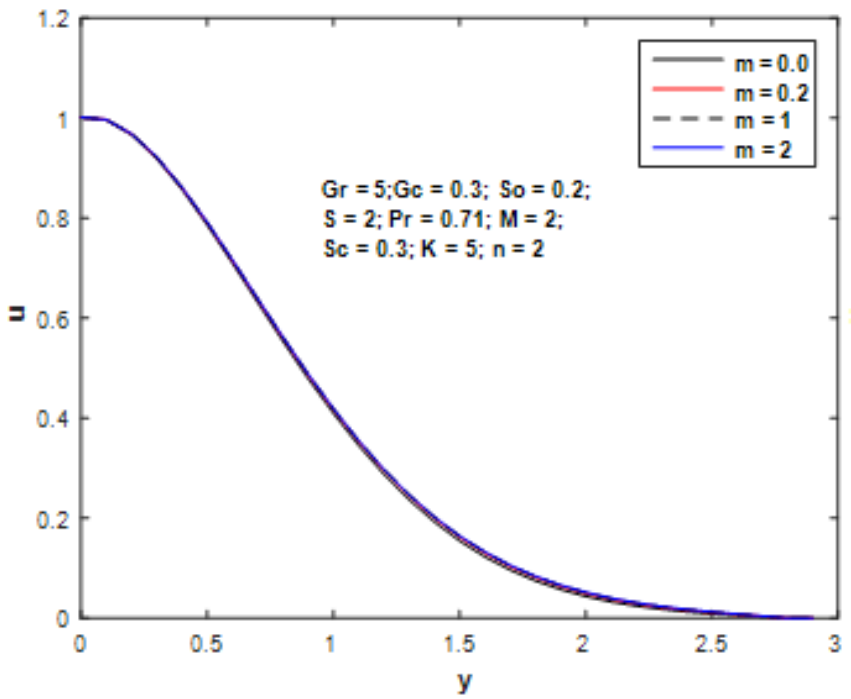

Figure 9: Variation on velocity field V/S $m$. 


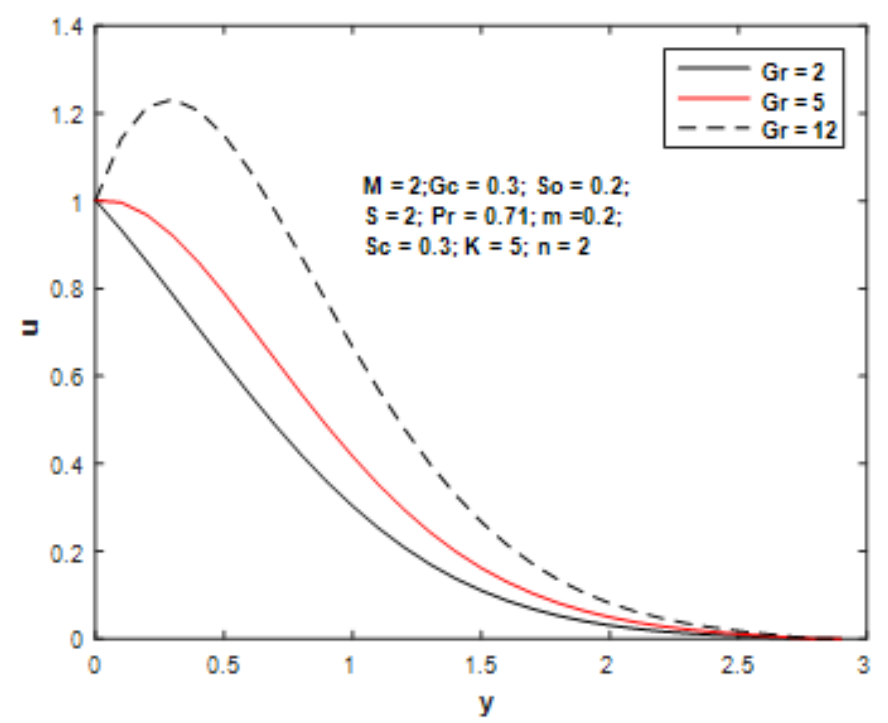

Figure 10: Variation on velocity field V/S Gr.

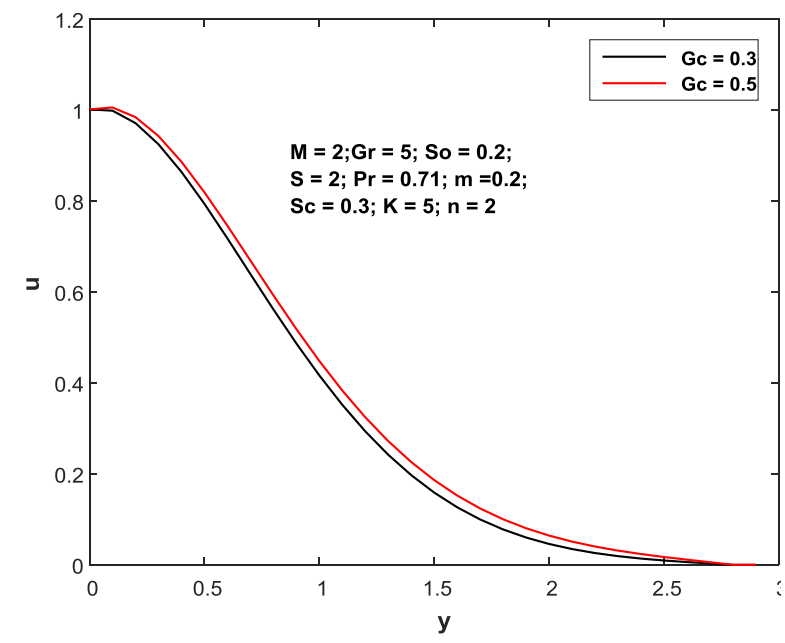

Figure 11: Changes on velocity field V/S Gc.

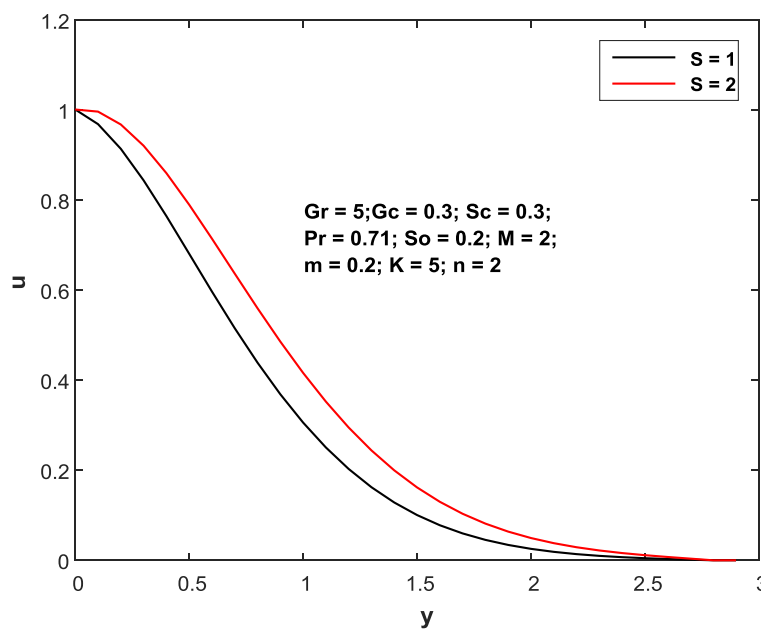

Figure 12: Variations $S$ V/S Velocity Field. 


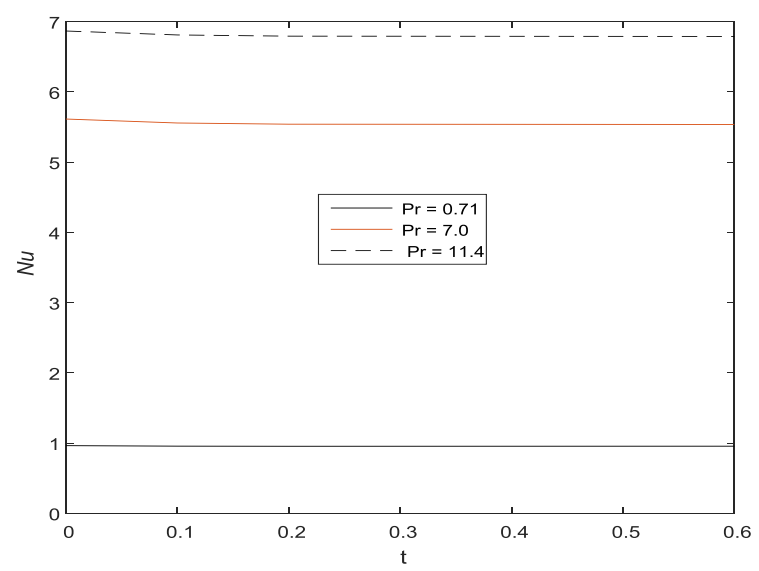

Figure 13: Changes on $n$ V/S Nusselt Number.

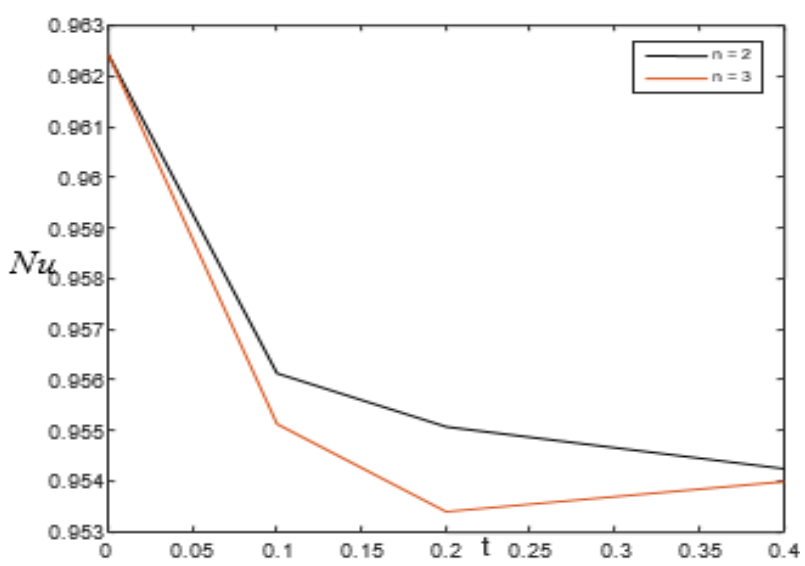

Figure 14: Changes on $\operatorname{Pr}$ V/S Nusselt Number.

\section{REFERENCES}

1. G. Dharmaiah, O.D. Makinde, and K.S. BalamuruganInt. Journal of Thermofluid Science and Technology7(1),2020,Paper No. 20070103.https://doi.org/10.36963/IJTST. 20070103.

2. Chamkha, A.J. Int. Comm. Heat Mass Transfer, V30, N3, 2003, pp. 413-422.

3. Kandasamy, R., Periasamy, K., andPrabhu, K.K.S. Int. J.Heat Mass Transfer, 48, 2005. pp. 4557-4561.

4. M.M. Rashidi, Computer Physics Comm., 180, 2009, pp.2210-2217.

5. Srihari, K., and ChirraKesava Reddy. "Effects of Soret and Magnetic Field on Unsteady Flow of a Radiating and Chemical Reacting Fluid: A Finite Difference Approach." International Journal of Mechanical Engineering 3.3 (2014).

6. L. Rama Mohan Reddy, M. C. Raju, andG. S. S. Raju, , Int. J. Appl. Comput. Math. 2016.

7. S. S. Das and M. Mitra, , Ind. J. Sci. Tech., 2 (5), pp.18-22, 2000.

8. S. S. Das, A. Satapathy, J. K. Das and J. P. Panda, Int. J. Heat Mass Transfer, 52, pp.5962-5969, 2009.

9. Mohammed Ibrahim S and Bhaskar Reddy N., Int. J of Appl. Math and Mech. 8(8), pp.1-21, 2012.

10. B. K. Jha, R. Prasad and S. Rai, .Astrophysics and Space Science181, pp.125-134, 1991.

11. Poonia, Hemant, and R. C. Chaudhary. "Mass transfer with chemical reaction effects on MHD free convective flow past an accelerated vertical plate embedded in a porous medium." Int. J. of Applied Mathematics \& Statistical Sciences 5 (2016). 
12. E. M. A. Elbashbeshy, D. M. Yassmin and A. A. Dalia, African Journal of Mathematics and Computer Science Research Vol. 3, no.5, pp. 68-73, 2010.

13. B. Shankar Goud., and M.N. Raja Shekar.,InternationalJournal of Computational and Applied Mathematics, 12(1), pp. 53-64, 2017.

14. Nasrawi, HayderKraidi Rashid. "Natural Convection Heat Transfer inside an Inclined Square Enclosure Filled with Al2o3 Nanofluid in Presence of Pair of Discrete Heat Flux Sources in Bottom Wall." International Journal of Mechanical Engineering (IJME) 3.1 (2014): 35-46.

15. Ali Chamkha, M.A and Mansour,A. A.Int. J. for Numerical Methods in Fluids, 65(4),pp.432-447, 2011.

16. Satya Narayana, P.V., Rami Reddy, G. and Venkataramana, S. , Int. J.of Automotive and Mechanical Engineering, 3, pp.350363, 2011.

17. Nandkeolyar, R., M. Das and P. Sibanda,,Boundary Value Problems, pp.1-16, 2013.

18. Srinivasa Raju R, Sudhakar K and Rangamma M. , Journal of Institution of Engineers (India): SeriesC. 94(2), pp. 175-186, 2013.

19. Magotra, Ankit, Anika Malik, and B. L Pander. "Candidate Genetic Markers Associated with Thermo-Tolerance in Animals." International Journal of Applied and Natural Sciences (IJANS) 6.6 (2017): 61-72.

20. B. Shankar Goud, B. Suresh Babu, MN Raja Shekar, and G.Srinivas, Int.l Journal of Thermofluid Science and Technology,6(4), 2019, Paper No. 19060402 https://doi.org/10.36963/IJTST.19060402.

21. B. Shankar Goud Inte. Journal of Thermofluid Science and Technology,7,(2),2020 Paper No.070201 https://doi.org/10.36963/IJTST.2020070201.

22. B. ShankarGoud, D Mahendar and M. N. Raja Shekar.,AIP Conference Proceedings 2246, 020081 (2020); https://doi.org/10.1063/5.0014524 